\title{
Quadratic Spline Quasi-interpolants on Powell-Sabin Partitions
}

\author{
Carla Manni and Paul Sablonnière
}

\begin{abstract}
In this paper we address the problem of constructing quasi-interpolants in the space of quadratic Powell-Sabin splines on nonuniform triangulations. Quasi-interpolants of optimal approximation order are proposed and numerical tests are presented.
\end{abstract}

Keywords: Quasi-interpolation, Quadratic Splines, Powell-Sabin refinement, Bézier-Bernstein representation.

AMS Subject classification: 65D05, 41A05, 41A25, 41A50.

\section{$\S 1 . I n t r o d u c t i o n$}

It is well known that the term quasi-interpolation denotes a general approach to construct, with low computational cost, efficient local approximants to a given set of data or a given function. A quasi-interpolant (q.i.) for a given function $f$ is usually obtained as linear combination of the elements of a suitable set of functions which are required to be positive, to ensure stability, and to have small local support in order to achieve local control. The coefficients of the linear combination are the values of linear functionals depending on $f$ and on its derivatives/integrals.

Quasi-interpolation has received a considerable attention by many authors since the seminal paper [18].

In the univariate case various effective quasi-interpolating schemes are available, based on values of $f$, and/or its derivatives, and/or its integrals (see for examples [1], [4], [12], [16], [18] and references quoted therein) and interesting applications have been proposed in different fields.

Similarly, various interesting results have been obtained in the bivariate setting by using quasi-interpolating schemes based on tensor-product polynomial splines (see for example [9], [16] and references quoted therein) or on spaces of splines over tridirectional and four directional partitions both for the uniform and the non-uniform case ([3], [5], [17]).

On the other hand, quasi-interpolation for splines over arbitrary triangulations seems to be a rather few explored field. 
In this paper we address the problem of constructing quasi-interpolants in the space of quadratic splines over a Powell-Sabin refinement of a generic triangulation, [14]. The low degree and the simple Bézier-Bernstein representation coupled with the possibility of handling arbitrary triangulations of polygonal domains, make this spline space very interesting not only from the theoretical point of view but also for applications. Thus, PowellSabin quadratic splines have been widely studied by several authors (see for example [2], [7], [8], [11], [13], [15], [19], [21], and references quoted therein).

In the space of Powell-Sabin quadratic splines the Hermite interpolation problem at the vertices of the triangulation has unique solution and the interpolant can be locally computed in each triangle of the triangulation. So, the Hermite interpolant can be seen as a special quasiinterpolant. However, other interesting quasi-interpolanting schemes can be constructed in this space. In particular we describe quasi-interpolants, not requiring derivatives of the function $f$, which reproduce quadratic polynomials and therefore provide the maximum approximation order in the space.

The results we present are based on the properties of the quadratic Powell-Sabin B-splines constructed and analyzed by Diercx and some coauthors, see [6], [19], [20], [21].

The remaining of the paper is divided into 4 sections. In the next one we briefly recall from [6] the construction and salient properties of quadratic B-splines over a Powell-Sabin refinement of a triangulation of a planar domain. In Section 3 we discuss differential quasi-interpolants and in Section 4 we construct two families of discrete quasi-interpolants reproducing bivariate quadratic polynomials. Finally, we end in Section 5 with some computed examples.

\section{§2. Quadratic B-spline over a Powell-Sabin refinement}

In this section we briefly summarize from [6] the construction and some properties of quadratic B-splines over a Powell-Sabin refinement of a triangulation of a planar domain (see also [19], [20], [21]).

For the sake of simplicity, in the following the Bézier-Bernstein representation will be used to describe polynomials over triangles (see for example [6], [14], [15]). Let $\tau$ be a triangle with vertices

$$
\mathbf{V}_{i_{j}}:=\left(x_{i_{j}}, y_{i_{j}}\right), j=1,2,3,
$$

and let $(u, v, w)$ be the barycentric coordinates of a point $(x, y) \in \mathbb{R}^{2}$ with respect to the triangle $\tau$, that is the values determined by the linear system

$$
\left(\begin{array}{ccc}
1 & 1 & 1 \\
x_{i_{1}} & x_{i_{2}} & x_{i_{3}} \\
y_{i_{1}} & y_{i_{2}} & y_{i_{3}}
\end{array}\right)\left(\begin{array}{c}
u \\
v \\
w
\end{array}\right)=\left(\begin{array}{c}
1 \\
x \\
y
\end{array}\right) .
$$


Let $\mathbb{P}_{n}$ denote the linear space of algebraic polynomials of degree less than ore equal to $n$. Any element $p \in \mathbb{P}_{n}$ has a unique representation in barycentric coordinates

$$
p(x, y)=\sum_{i+j+k=n} b_{i, j, k} \frac{n !}{i ! j ! k !} u^{i} v^{j} w^{k} .
$$

The coefficients $b_{i, j, k}$ are the Bézier ordinates of the polynomial $p$ with respect to the triangle $\tau$. Usually, this representation is called BézierBernstein representation of $p$ and it is schematically represented by associating each coefficient $b_{i, j, k}$ with the point

$$
\left(x_{i, j, k}, y_{i, j, k}\right)
$$

having barycentric coordinates $\left(\frac{i}{n}, \frac{j}{n}, \frac{k}{n}\right)$. The points

$$
\left(x_{i, j, k}, y_{i, j, k}, b_{i, j, k}\right) \in \mathbb{R}^{3}, i+j+k=n,
$$

are the Bézier control points of $p$.
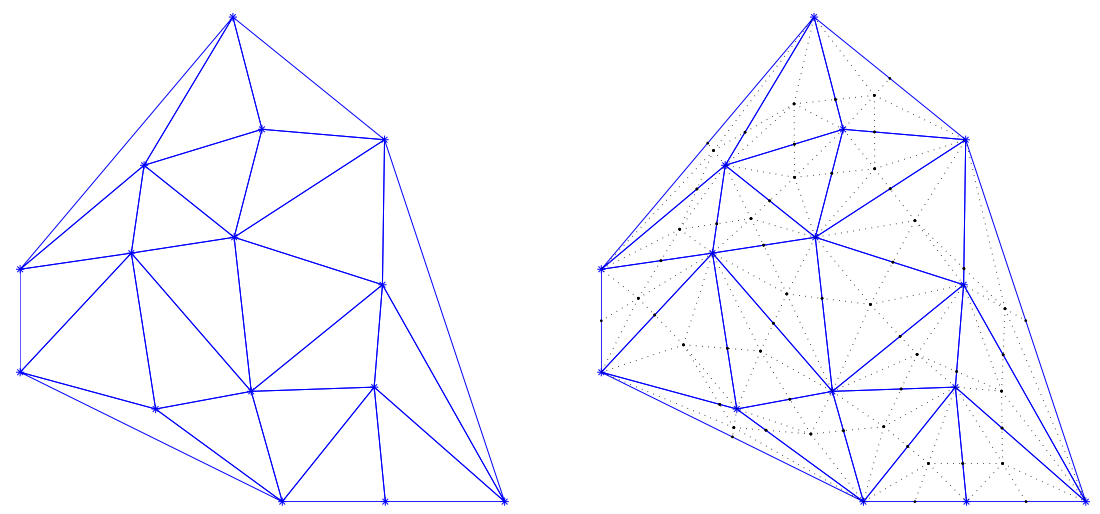

Fig. 1. A triangulation $\Delta$ and a Powell-Sabin refinement $\Delta_{P S}$ of $\Delta$.

Let $\Omega$ be a polygonal domain in $\mathbb{R}^{2}$ and let $\Delta$ be a conforming (1) triangulation of $\Omega$. We denote by

$$
\mathbf{V}_{l}:=\left(x_{l}, y_{l}\right), l=1, \ldots, N_{v}
$$

the vertices of the given triangulation. A Powell-Sabin refinement, $\Delta_{P S}$, of $\Delta$ is the refined triangulation, [14], obtained (see Fig.1) by subdividing each triangle of $\Delta$ into six subtriangles as follows. Select a point, say $\mathbf{C}_{j}$,

(1) $\Delta$ is a conforming triangulation if any edge of a triangle $\tau_{l}$ does not contain any vertex of anther triangle $\tau_{r}$ different from its own two vertices. 
inside any triangle $\tau_{j}$ of $\Delta$ and connect it with the three vertices of $\tau_{j}$ and with the points $\mathbf{C}_{j_{1}}, \mathbf{C}_{j_{2}}, \mathbf{C}_{j_{3}}$ where $\tau_{j_{1}}, \tau_{j_{2}}, \tau_{j_{3}}$ are the triangles adjacent to $\tau_{j}{ }^{(2)}$. We assume that each segment $\mathbf{C}_{j} \mathbf{C}_{j_{i}}, i=1,2,3$, intersects the interior of the common edge of $\tau_{j}$ and $\tau_{j_{i}}$.

We denote by $\mathcal{S}_{2}^{1}\left(\Delta_{P S}\right)$ the space of Powell-Sabin splines, [14], that is the linear space of piecewise quadratic polynomials on $\Delta_{P S}$ with $C^{1}$ continuity in $\Omega$. It is well known, [6], [14], [15], that:

- $\operatorname{dim}\left(\mathcal{S}_{2}^{1}\left(\Delta_{P S}\right)\right)=3 N_{v}$

- any element of $\mathcal{S}_{2}^{1}\left(\Delta_{P S}\right)$ is determined by its value and its gradient at the vertices of $\Delta$;

- any element of $\mathcal{S}_{2}^{1}\left(\Delta_{P S}\right)$ can be locally computed in each triangle of $\Delta$ provided that its values and its gradients at the three vertices of the triangle are given.

In [6] $B$-spline bases of the space $\mathcal{S}_{2}^{1}\left(\Delta_{P S}\right)$ have been proposed as follows. Let us associate three functions to any vertex of $\Delta$

$$
\left\{B_{l}^{(j)}, \quad j=1,2,3, \quad l=1, \ldots, N_{v}\right\},
$$

such that

$$
\begin{aligned}
& s=\sum_{l=1}^{N_{v}} \sum_{j=1}^{3} c_{l, j} B_{l}^{(j)}, \forall s \in \mathcal{S}_{2}^{1}\left(\Delta_{P S}\right), \\
& B_{l}^{(j)}(x, y) \geq 0, \\
& \sum_{l=1}^{N_{v}} \sum_{j=1}^{3} B_{l}^{(j)}(x, y)=1 .
\end{aligned}
$$

The functions $B_{l}^{(j)}$ will be referred to as Powell-Sabin B-splines.

From the Bézier-Bernstein representation, it is immediate to see that the smallest support for any $B_{l}^{(j)}$ is the cell $\Delta_{l}$, that is the union of all triangles of $\Delta$ containing the vertex $\mathbf{V}_{l}$ (see Fig. 2).

The three functions $B_{l}^{(1)}, B_{l}^{(2)}, B_{l}^{(3)}$ can be locally constructed over the cell $\Delta_{l}$ once their values and gradients at any vertex of $\Delta$ are given. Due to the structure of the support $\Delta_{l}$ we have:

$$
B_{l}^{(j)}\left(\mathbf{V}_{k}\right)=0, \frac{\partial}{\partial x} B_{l}^{(j)}\left(\mathbf{V}_{k}\right)=0, \frac{\partial}{\partial y} B_{l}^{(j)}\left(\mathbf{V}_{k}\right)=0, \text { if } k \neq l .
$$

Moreover, we denote:

$$
B_{l}^{(j)}\left(\mathbf{V}_{l}\right)=: \alpha_{l}^{(j)}, \quad \frac{\partial}{\partial x} B_{l}^{(j)}\left(\mathbf{V}_{l}\right)=: \beta_{l}^{(j)}, \quad \frac{\partial}{\partial y} B_{l}^{(j)}\left(\mathbf{V}_{l}\right)=: \gamma_{l}^{(j)}
$$

(2) If $\tau_{j}$ is a boundary triangle the undefined $\mathbf{C}_{j_{i}}$ are specified points (usually the midpoints) over the corresponding boundary edges. 




Fig. 2. The cell $\Delta_{l}$ for the interior vertex $V_{l}$.

In order to obtain a partition of unity, we have to impose

$$
\begin{gathered}
\alpha_{l}^{(1)}+\alpha_{l}^{(2)}+\alpha_{l}^{(3)}=1, \\
\beta_{l}^{(1)}+\beta_{l}^{(2)}+\beta_{l}^{(3)}=0, \\
\gamma_{l}^{(1)}+\gamma_{l}^{(2)}+\gamma_{l}^{(3)}=0 .
\end{gathered}
$$

Using Bernstein-Bézier representation, it is possible to prove, [6], that $B_{l}^{(j)}$ is non negative if and only if the Bézier ordinates of the Bézier points "surrounding" the vertex $\mathbf{V}_{l}$ are non negative (see Fig. 3). The positivity of the above mentioned coefficients has the following very nice geometric interpretation, [6]. Let us denote $h(x, y)=x, g(x, y)=y$. For each index $l$, we want to determine points $\left\{\mathbf{Q}_{l}^{(j)}, j=1,2,3\right\}$ such that the following expansions hold

$$
h=\sum_{l=1}^{N_{v}} \sum_{j=1}^{3} h\left(\mathbf{Q}_{l}^{(j)}\right) B_{l}^{(j)}, \quad g=\sum_{l=1}^{N_{v}} \sum_{j=1}^{3} g\left(\mathbf{Q}_{l}^{(j)}\right) B_{l}^{(j)} .
$$

Since $h, g \in \mathcal{S}_{2}^{1}\left(\Delta_{P S}\right)$, then they are uniquely determined by their values and their gradients at the vertices of $\Delta$. So, denoting by $A_{k, x}, A_{k, y}$ the coordinates of the point $\mathbf{A}_{k}$, we have

$$
M_{l}\left(\begin{array}{c}
Q_{l, x}^{(1)} \\
Q_{l, x}^{(2)} \\
Q_{l, x}^{(3)}
\end{array}\right)=\left(\begin{array}{c}
x_{l} \\
1 \\
0
\end{array}\right), M_{l}\left(\begin{array}{c}
Q_{l, y}^{(1)} \\
Q_{l, y}^{(2)} \\
Q_{l, y}^{(3)}
\end{array}\right)=\left(\begin{array}{c}
y_{l} \\
0 \\
1
\end{array}\right), \quad l=1, \ldots, N_{v}
$$

where

$$
M_{l}:=\left(\begin{array}{ccc}
\alpha_{l}^{(1)} & \alpha_{l}^{(2)} & \alpha_{l}^{(3)} \\
\beta_{l}^{(1)} & \beta_{l}^{(2)} & \beta_{l}^{(3)} \\
\gamma_{l}^{(1)} & \gamma_{l}^{(2)} & \gamma_{l}^{(3)}
\end{array}\right)
$$




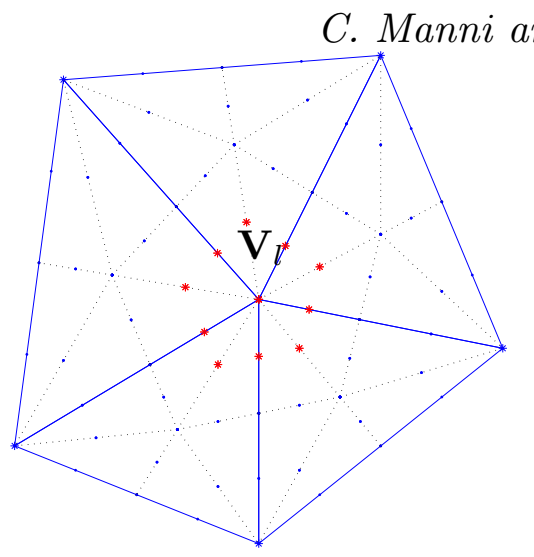

Fig. 3. *: location of the Bézier coefficients "surrounding" the vertex $V_{l}$.

The B-splines are required to be linearly independent, therefore the matrix $M_{l}$ is non singular. So, for each vertex $\mathbf{V}_{l}$, systems (7) uniquely determine three points $\mathbf{Q}_{l}^{(j)}, j=1,2,3$. From [6], Section 4, we have the following result (see also Fig. 4 and Fig. 5)

Theorem 1. The functions $B_{l}^{(j)}, j=1,2,3$, are non negative if and only if the triangle with vertices $\mathbf{Q}_{l}^{(j)}, j=1,2,3$, contains the Bézier points (1) surrounding $\mathbf{V}_{l}$.
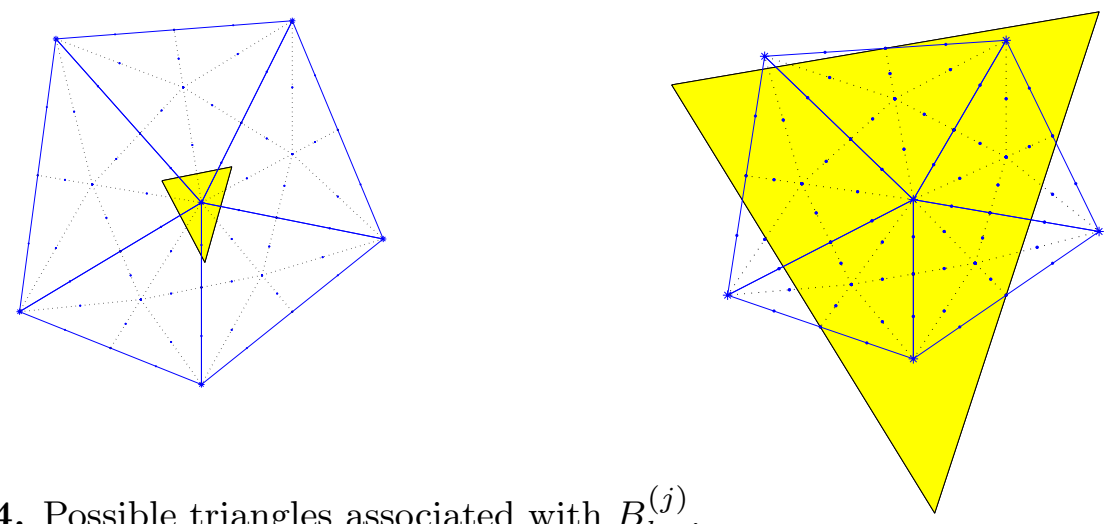

Fig. 4. Possible triangles associated with $B_{l}^{(j)}$.

On the other hand, it is immediate to see that, given three non collinear points $\mathbf{Q}_{l}^{(j)}, j=1,2,3$, they uniquely determine, via (7) and (5), the values and gradients of the three functions $B_{l}^{(j)}, j=1,2,3$, at the vertex $\mathbf{V}_{l}$ and so they completely determine the three functions $B_{l}^{(j)}$. Summarizing, the points $\mathbf{Q}_{l}^{(j)}, j=1,2,3$ - and so the triangle they form - are uniquely associated with the triple $B_{l}^{(j)}, j=1,2,3$, and can be efficiently used to identify and describe these functions and their properties instead of $\alpha_{l}^{(j)}, \beta_{l}^{(j)}, \gamma_{l}^{(j)}$. Moreover, [6], triangles with a small area produce B-splines with "better" computational properties ("more pronounced 

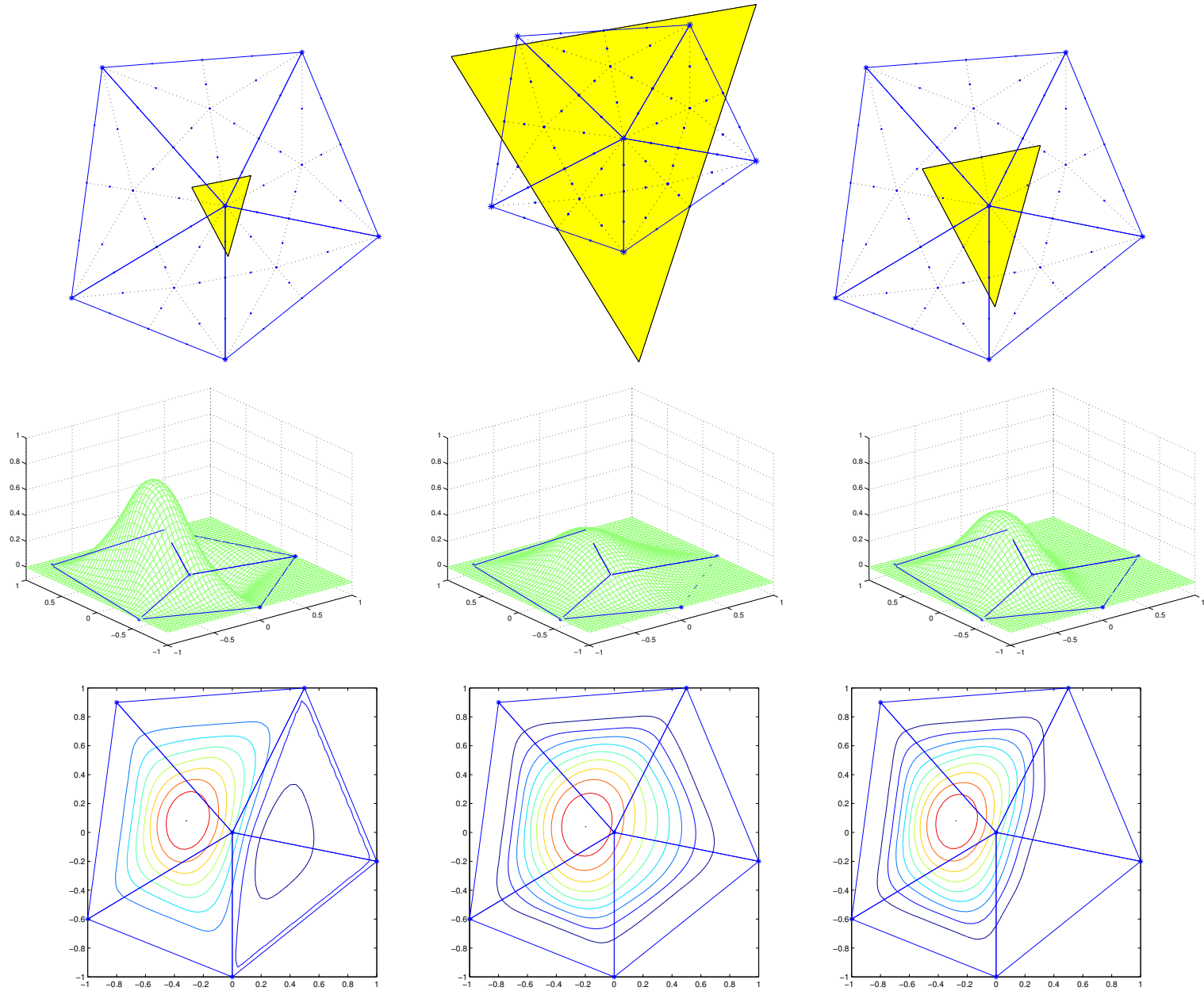

Fig. 5. Possible triangles associated with $B_{l}^{(j)}$ and their effect on the positivity and the shape of the B-splines. Top to bottom: the triangles, the associated function $B_{l}^{(1)}$, contour lines for $B_{l}^{(1)}$.

linear independence" taking into account positivity, see (7) and Fig. 5). In [6] an optimization strategy has been proposed to select triangles with minimal area ensuring positivity of the corresponding B-splines.

\section{$\S$ 3. Differential Quasi-interpolants in $\mathcal{S}_{2}^{1}\left(\Delta_{P S}\right)$}

In this section we describe how the B-splines introduced in Section 2 can be used to define quasi-interpolants, based on derivatives of the given function $f$, which are exact on $\mathbb{P}_{2}$ and provide the best approximation order in the space $\mathcal{S}_{2}^{1}\left(\Delta_{P S}\right)$. 
We are interested in quasi-interpolants of the following form

$$
\mathcal{Q} f=\sum_{l=1}^{N_{v}} \sum_{j=1}^{3} \mu_{l}^{(j)}(f) B_{l}^{(j)},
$$

where $\mu_{l}^{(j)}(f), j=1,2,3, l=1, \ldots, N_{v}$, are suitable linear functionals. First of all we note (see also [20]) that, if

$$
f\left(\mathbf{V}_{l}\right), \quad \nabla f\left(\mathbf{V}_{l}\right), \quad l=1, \ldots, N_{v}
$$

are given, setting

$$
\left(\begin{array}{c}
\mu_{l}^{(1)}(f) \\
\mu_{l}^{(2)}(f) \\
\mu_{l}^{(3)}(f)
\end{array}\right):=M_{l}^{-1}\left(\begin{array}{c}
f\left(\mathbf{V}_{l}\right) \\
f_{x}\left(\mathbf{V}_{l}\right) \\
f_{y}\left(\mathbf{V}_{l}\right)
\end{array}\right)
$$

where $M_{l}$ is defined in (8), then, from (4), expression (9) provides the unique element in $\mathcal{S}_{2}^{1}\left(\Delta_{P S}\right)$ which interpolates the data (10). So, the scheme (9) with coefficients given by (11) is a quasi-interpolating (actually interpolating) scheme in $\mathcal{S}_{2}^{1}\left(\Delta_{P S}\right)$ which obviously reproduces $\mathbb{P}_{2}$. Moreover, (11) and (8) show that this is the unique quasi-interpolant of the form (9) with

$$
\mu_{l}^{(j)}(f):=a_{l}^{(j)} f\left(\mathbf{V}_{l}\right)+b_{l}^{(j)} f_{x}\left(\mathbf{V}_{l}\right)+c_{l}^{(j)} f_{y}\left(\mathbf{V}_{l}\right), \quad a_{l}^{(j)}, b_{l}^{(j)}, c_{l}^{(j)} \in \mathbb{R},
$$

which is a projection, [10], in $\mathcal{S}_{2}^{1}\left(\Delta_{P S}\right)$, i.e. satisfies

$$
\mathcal{Q} s=s, \quad \forall s \in \mathcal{S}_{2}^{1}\left(\Delta_{P S}\right) .
$$

A second quasi-interpolant reproducing $\mathbb{P}_{2}$ can be obtained as a modification of the scheme

$$
\mathcal{Q}_{1} f:=\sum_{l=1}^{N_{v}} \sum_{j=1}^{3} f\left(\mathbf{Q}_{l}^{(j)}\right) B_{l}^{(j)} .
$$

From (5) and (6) we have

$$
\left(\mathcal{Q}_{1} p\right)\left(\mathbf{V}_{l}\right)=p\left(\mathbf{V}_{l}\right), \nabla\left(\mathcal{Q}_{1} p\right)\left(\mathbf{V}_{l}\right)=\nabla p\left(\mathbf{V}_{l}\right), l=1, \ldots, N_{v}, \forall p \in \mathbb{P}_{1},
$$

so that

$$
\mathcal{Q}_{1} p=p, \quad \forall p \in \mathbb{P}_{1} .
$$

Thus, we modify $\mathcal{Q}_{1} f$ in order to obtain reproduction of quadratic polynomials. We have the following result 
Theorem 2. Let us define

$$
\tilde{\mathcal{Q}}_{2} f:=\sum_{l=1}^{N_{v}} \sum_{j=1}^{3} \tilde{\mu}_{l}^{(j)}(f) B_{l}^{(j)} .
$$

where, for $j=1,2,3, l=1, \ldots, N_{v}$

$$
\tilde{\mu}_{l}^{(j)}(f):=f\left(\mathbf{Q}_{l}^{(j)}\right)-\frac{1}{2}\left(\mathbf{Q}_{l}^{(j)}-\mathbf{V}_{l}\right) \nabla^{2} f\left(\mathbf{V}_{l}\right)\left(\mathbf{Q}_{l}^{(j)}-\mathbf{V}_{l}\right)^{T}
$$

Then,

$$
\tilde{\mathcal{Q}}_{2} p=p, \forall p \in \mathbb{P}_{2}
$$

Proof: Let us consider $p \in \mathbb{P}_{2}$. From Taylor expansion

$p\left(\mathbf{Q}_{l}^{(j)}\right)=p\left(\mathbf{V}_{l}\right)+\nabla p\left(\mathbf{V}_{l}\right)\left(\mathbf{Q}_{l}^{(j)}-\mathbf{V}_{l}\right)^{T}+\frac{1}{2}\left(\mathbf{Q}_{l}^{(j)}-\mathbf{V}_{l}\right) \nabla^{2} p\left(\mathbf{V}_{l}\right)\left(\mathbf{Q}_{l}^{(j)}-\mathbf{V}_{l}\right)^{T}$

thus, from (15)

$$
\tilde{\mathcal{Q}}_{2} p=\sum_{i=1}^{N_{v}} \sum_{j=1}^{3}\left[p\left(\mathbf{V}_{i}\right)+\nabla p\left(\mathbf{V}_{i}\right)\left(\mathbf{Q}_{i}^{(j)}-\mathbf{V}_{i}\right)^{T}\right] B_{i}^{(j)}
$$

Hence, from (3)

$$
\begin{aligned}
& \left(\tilde{Q}_{2} p\right)\left(\mathbf{V}_{l}\right)=\sum_{j=1}^{3}\left[p\left(\mathbf{V}_{l}\right)+\nabla p\left(\mathbf{V}_{l}\right)\left(\mathbf{Q}_{l}^{(j)}-\mathbf{V}_{l}\right)^{T}\right] B_{l}^{(j)}\left(\mathbf{V}_{l}\right)= \\
& p\left(\mathbf{V}_{l}\right) \sum_{j=1}^{3} B_{l}^{(j)}\left(\mathbf{V}_{l}\right)+\nabla p\left(\mathbf{V}_{l}\right)\left(\sum_{j=1}^{3} \mathbf{Q}_{l}^{(j)} B_{l}^{(j)}\left(\mathbf{V}_{l}\right)\right)^{T}-\nabla p\left(\mathbf{V}_{l}\right)\left(\mathbf{V}_{l}\right)^{T} \sum_{j=1}^{3} B_{l}^{(j)}\left(\mathbf{V}_{l}\right),
\end{aligned}
$$

so that, from (4), (5) and (7) we obtain

$$
\left(\tilde{\mathcal{Q}}_{2} p\right)\left(\mathbf{V}_{l}\right)=p\left(\mathbf{V}_{l}\right), l=1, \ldots, N_{v}
$$

Similarly,

$$
\nabla\left(\tilde{\mathcal{Q}}_{2} p\right)\left(\mathbf{V}_{l}\right)=\nabla p\left(\mathbf{V}_{l}\right), l=1, \ldots, N_{v} .
$$

The assertion follows since any element in $\mathcal{S}_{2}^{1}\left(\Delta_{P S}\right)$ is uniquely determined by its values and gradients at the vertices of $\Delta$. 


\section{$\S$ 4. Discrete Quasi-interpolants in $\mathcal{S}_{2}^{1}\left(\Delta_{P S}\right)$}

The Hermite interpolant and the q.i. (14) require the computation of values of the function $f$ and of some of its derivatives. In this section we describe how it is possible to construct quasi-interpolants of the form (9) which reproduce $\mathbb{P}_{2}$ and do not require values of derivatives of $f$. More precisely, we are interested in quasi-interpolants of the form (9) with

$$
\begin{gathered}
\mu_{l}^{(j)}(f):=q_{l}^{(j, 1)} f\left(\mathbf{Z}_{l}^{(j, 1)}\right)+q_{l}^{(j, 2)} f\left(\mathbf{Z}_{l}^{(j, 2)}\right)+q_{l}^{(j, 3)} f\left(\mathbf{Z}_{l}^{(j, 3)}\right) \\
q_{l}^{(j, 1)}, q_{l}^{(j, 2)}, q_{l}^{(j, 3)} \in \mathbb{R}, \quad \mathbf{Z}_{l}^{(j, 1)}, \mathbf{Z}_{l}^{(j, 2)}, \mathbf{Z}_{l}^{(j, 3)} \in \mathbb{R}^{2} .
\end{gathered}
$$

Since the set of functions (2) forms a basis of $\mathcal{S}_{2}^{1}\left(\Delta_{P S}\right)$, from (12) and (13) we deduce that any q.i. defined via (9) and (16) reproduces $\mathbb{P}_{1}$ if and only if the scalars $q_{l}^{(j, 1)}, q_{l}^{(j, 2)}, q_{l}^{(j, 3)}$ are the barycentric coordinates of $\mathbf{Q}_{l}^{(j)}$ with respect to the triple of points $\mathbf{Z}_{l}^{(j, 1)}, \mathbf{Z}_{l}^{(j, 2)}, \mathbf{Z}_{l}^{(j, 3)}$.

The following result shows that the triple of points $\mathbf{Z}_{l}^{(j, k)}, k=1,2,3$, for a q.i. of the form (9) and (16) which reproduces quadratic polynomials have a specific geometric configuration.

Theorem 3. Let $\mathcal{Q}$ be any q.i. of the form (9) with $\mu_{l}^{(j)}$ defined according to (16). For $l=1, \ldots, N_{v}, j=1,2,3$, let us define the set of indices

$$
\mathcal{I}_{l}^{(j)}:=\left\{k \text { such that } q_{l}^{(j, k)} \neq 0\right\}
$$

If

$$
\mathcal{Q} p=p, \quad \forall p \in \mathbb{P}_{2}
$$

then, for any $l \in\left\{1, \ldots, N_{v}\right\}$ and $j \in\{1,2,3\}$ the points

$$
\mathbf{Q}_{l}^{(j)}, \mathbf{V}_{l}, \text { and }\left\{\mathbf{Z}_{l}^{(j, k)}, k \in \mathcal{I}_{l}^{(j)}\right\}
$$

are collinear.

Proof: From Theorem 2 we know that (15) provides the coefficients of the representation of any polynomial of degree less than or equal to 2 with respect to the basis (2). Thus, from (17) we have that

$$
\mu_{l}^{(j)}(p)=\tilde{\mu}_{l}^{(j)}(p), \quad \forall p \in \mathbb{P}_{2}, l=1, \ldots, N_{v}, \quad j=1,2,3
$$

Let us fix $l \in\left\{1, \ldots, N_{v}\right\}$ and $j \in\{1,2,3\}$. Without loss of generality we can assume $\mathbf{V}_{l}=(0,0)$. Specifying equality (18) for the monomial basis 
of bivariate quadratic polynomials we obtain

$$
\begin{aligned}
q_{l}^{(j, 1)}+q_{l}^{(j, 2)}+q_{l}^{(j, 3)} & =1 \\
q_{l}^{(j, 1)} Z_{l, x}^{(j, 1)}+q_{l}^{(j, 2)} Z_{l, x}^{(j, 2)}+q_{l}^{(j, 3)} Z_{l, x}^{(j, 3)} & =Q_{l, x}^{(j)} \\
q_{l}^{(j, 1)} Z_{l, y}^{(j, 1)}+q_{l}^{(j, 2)} Z_{l, y}^{(j, 2)}+q_{l}^{(j, 3)} Z_{l, y}^{(j, 3)} & =Q_{l, y}^{(j)} \\
q_{l}^{(j, 1)}\left(Z_{l, x}^{(j, 1)}\right)^{2}+q_{l}^{(j, 2)}\left(Z_{l, x}^{(j, 2)}\right)^{2}+q_{l}^{(j, 3)}\left(Z_{l, x}^{(j, 3)}\right)^{2} & =0 \\
q_{l}^{(j, 1)}\left(Z_{l, y}^{(j, 1)}\right)^{2}+q_{l}^{(j, 2)}\left(Z_{l, y}^{(j, 2)}\right)^{2}+q_{l}^{(j, 3)}\left(Z_{l, y}^{(j, 3)}\right)^{2} & =0 \\
q_{l}^{(j, 1)} Z_{l, x}^{(j, 1)} Z_{l, y}^{(j, 1)}+q_{l}^{(j, 2)} Z_{l, x}^{(j, 2)} Z_{l, y}^{(j, 2)}+q_{l}^{(j, 3)} Z_{l, x}^{(j, 3)} Z_{l, y}^{(j, 3)} & =0
\end{aligned}
$$

It is clear that system (19) can have solutions only if the rows of the matrix

$$
\left(\begin{array}{ccc}
\left(Z_{l, x}^{(j, 1)}\right)^{2} & \left(Z_{l, x}^{(j, 2)}\right)^{2} & \left(Z_{l, x}^{(j, 3)}\right)^{2} \\
\left(Z_{l, y}^{(j, 1)}\right)^{2} & \left(Z_{l, y}^{(j, 2)}\right)^{2} & \left(Z_{l, y}^{(j, 3)}\right)^{2} \\
Z_{l, x}^{(j, 1)} Z_{l, y}^{(j, 1)} & Z_{l, x}^{(j, 2)} Z_{l, y}^{(j, 2)} & Z_{l, x}^{(j, 3)} Z_{l, y}^{(j, 3)}
\end{array}\right)
$$

are linearly dependent, that is only if the cartesian coordinates of the points $\mathbf{Z}_{l}^{(j, 1)}, \mathbf{Z}_{l}^{(j, 2)}, \mathbf{Z}_{l}^{(j, 3)}$ verify the equation

$$
r x^{2}+s y^{2}+t x y=0
$$

for some $r, s, t \in \mathbb{R}, r^{2}+s^{2}+t^{2}>0$. The locus determined by (20) in the plane is the product of two straight lines through the origin or reduces to the point $(0,0)$. Thus, if system (19) has solutions the points $\mathbf{Z}_{l}^{(j, 1)}, \mathbf{Z}_{l}^{(j, 2)}, \mathbf{Z}_{l}^{(j, 3)}$, belong to two straight lines through $\mathbf{V}_{l}$.

If $\mathbf{Z}_{l}^{(j, 1)}, \mathbf{Z}_{l}^{(j, 2)}, \mathbf{Z}_{l}^{(j, 3)}$ and $\mathbf{V}_{l}$ are collinear thus $\mathbf{Q}_{l}^{(j)}$ has to belong to the same line otherwise the first three equations of (19) can not hold.

If only two points, say $\mathbf{Z}_{l}^{(j, 1)}, \mathbf{Z}_{l}^{(j, 2)}$, and $\mathbf{V}_{l}$ are collinear then $q_{l}^{(j, 3)}=$ 0 (otherwise, from the last three equations in (19) $\mathbf{Z}_{l}^{(j, 3)}$ is collinear with $\mathbf{Z}_{l}^{(j, 1)}, \mathbf{Z}_{l}^{(j, 2)}$ and $\mathbf{V}_{l}$ ) thus $\mathbf{Q}_{l}^{(j)}$ belongs to the line through $\mathbf{Z}_{l}^{(j, 1)}, \mathbf{Z}_{l}^{(j, 2)}$, and $\mathbf{V}_{l}$.

Summarizing, the assertion holds for all the admissible configurations of the triple of points $\mathbf{Z}_{l}^{(j, 1)}, \mathbf{Z}_{l}^{(j, 2)}, \mathbf{Z}_{l}^{(j, 3)}$.

Now, considering the results of Theorem 3 we construct a family of quasi-interpolants of the form (9) with coefficients given by (16) reproducing quadratic polynomials.

Theorem 4. For $l=1, \ldots, N_{v}, j=1,2,3$ let $\mathbf{W}_{l_{j}} \in \mathbb{R}^{2}$ be so that

$$
\mathbf{Q}_{l}^{(j)}=\lambda_{l}^{(j)} \mathbf{V}_{l}+\left(1-\lambda_{l}^{(j)}\right) \mathbf{W}_{l_{j}}, \lambda_{l}^{(j)} \in \mathbb{R}
$$


Let $\mathcal{Q}$ be any q.i. of the form (9) with $\mu_{l}^{(j)}$ defined according to (16) where

$$
\begin{aligned}
& \mathbf{Z}_{l}^{(j, 1)}:=\mathbf{V}_{l}, \\
& \mathbf{Z}_{l}^{(j, 2)}:=\zeta_{l}^{(j)} \mathbf{V}_{l}+\left(1-\zeta_{l}^{(j)}\right) \mathbf{W}_{l_{j}}, \quad \zeta_{l}^{(j)} \in \mathbb{R}, \zeta_{l}^{(j)} \neq 0,1, \\
& \mathbf{Z}_{l}^{(j, 3)}:=\mathbf{W}_{l_{j}},
\end{aligned}
$$

and

$$
\begin{aligned}
q_{l}^{(j, 1)} & :=\frac{\lambda_{l}^{(j)}\left(2-\zeta_{l}^{(j)}\right)-1}{1-\zeta_{l}^{(j)}}, \\
q_{l}^{(j, 2)} & :=\frac{1-\lambda_{l}^{(j)}}{\zeta_{l}^{(j)}\left(1-\zeta_{l}^{(j)}\right)}, \quad l=1, \ldots, N_{v}, j=1,2,3, \\
q_{l}^{(j, 3)} & :=\frac{\left(\lambda_{l}^{(j)}-1\right)\left(1-\zeta_{l}^{(j)}\right)}{\zeta_{l}^{(j)}}
\end{aligned}
$$

then

$$
\mathcal{Q} p=p, \forall p \in \mathbb{P}_{2} \text {. }
$$

Proof: For any $l=1, \ldots, N_{v}$, and $j=1,2,3$, we consider the triple of points $\mathbf{Z}_{l}^{(j, k)}, k=1,2,3$, given in (21) and we impose

$$
\mu_{l}^{(j)}(p)=\tilde{\mu}_{l}^{(j)}(p), \quad p \in\left\{1, x, y, x^{2}, y^{2}, x y\right\} .
$$

This results (see also the proof of the Theorem 3) in the system

$$
\begin{aligned}
q_{l}^{(j, 1)}+q_{l}^{(j, 2)}+q_{l}^{(j, 3)} & =1 \\
\left(q_{l}^{(j, 1)}+\zeta_{l}^{(j)} q_{l}^{(j, 2)}\right) \mathbf{V}_{l}+\left[q_{l}^{(j, 2)}\left(1-\zeta_{l}^{(j)}\right)+q_{l}^{(j, 3)}\right] \mathbf{W}_{l_{j}} & =\lambda_{l}^{(j)} \mathbf{V}_{l}+\left(1-\lambda_{l}^{(j)}\right) \mathbf{W}_{l_{j}} \\
{\left[q_{l}^{(j, 2)}\left(1-\zeta_{l}^{(j)}\right)^{2}+q_{l}^{(j, 3)}\right]\left(W_{l_{j}, x}-V_{l, x}\right)^{2} } & =0 \\
{\left[q_{l}^{(j, 2)}\left(1-\zeta_{l}^{(j)}\right)^{2}+q_{l}^{(j, 3)}\right]\left(W_{l_{j}, y}-V_{l, y}\right)^{2} } & =0 \\
{\left[q_{l}^{(j, 2)}\left(1-\zeta_{l}^{(j)}\right)^{2}+q_{l}^{(j, 3)}\right]\left(W_{l_{j}, x}-V_{l, x}\right)\left(W_{l_{j}, y}-V_{l, y}\right) } & =0
\end{aligned}
$$

that is

$$
\begin{aligned}
q_{l}^{(j, 1)}+q_{l}^{(j, 2)}+q_{l}^{(j, 3)} & =1 \\
q_{l}^{(j, 1)}+\zeta_{l}^{(j)} q_{l}^{(j, 2)} & =\lambda_{l}^{(j)} \\
q_{l}^{(j, 2)}\left(1-\zeta_{l}^{(j)}\right)+q_{l}^{(j, 3)} & =1-\lambda_{l}^{(j)} \\
q_{l}^{(j, 2)}\left(1-\zeta_{l}^{(j)}\right)^{2}+q_{l}^{(j, 3)} & =0 .
\end{aligned}
$$

It is immediate to verify that the values (22) uniquelly solve (23) so that the corresponding $\mu_{l}^{(j)}(p), l=1, \ldots, N_{v}, j=1,2,3$, exactly provide the coefficients of $p \in \mathbb{P}_{2}$ with respect to the basis (2). 

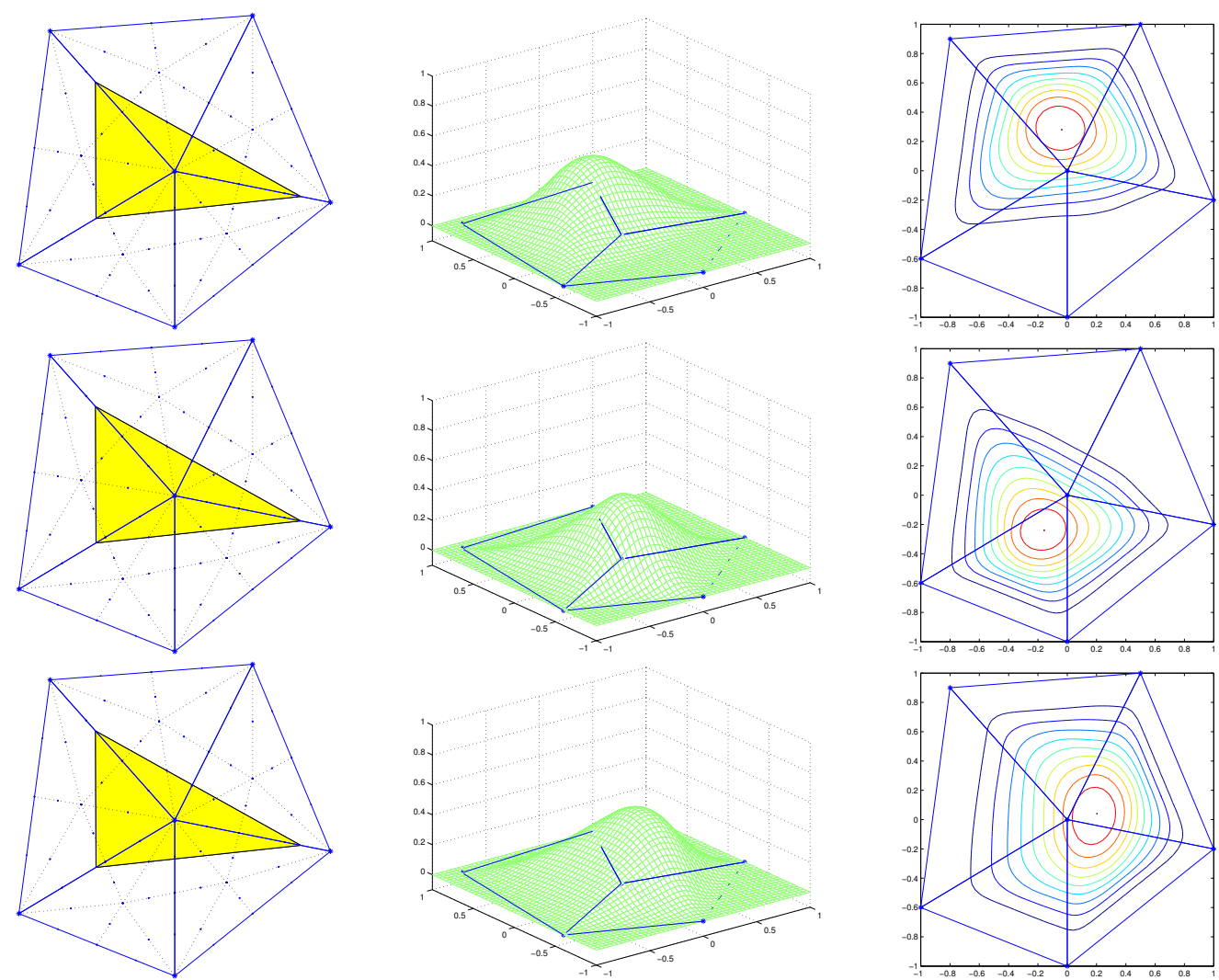

Fig. 6. The three B-splines, $B_{l}^{(j)}, j=1,2,3$ associated with the vertex $V_{l}$. Left to right: the determining triangle, the associated function $B_{l}^{(j)}$, contour lines for $B_{l}^{(j)}$.

The quasi-interpolants constructed in Theorem 4 require evaluating $f$ at points collinear with the vertices of $\Delta$ and with the points $\mathbf{Q}_{l}^{(j)}$ defining the used family of B-splines. So, it seems natural to investigate the case where the above mentioned points lie over the edges of $\Delta$. In order to do that we have to assume that the partition $\Delta$ and the family of B-splines we are dealing with verify the following hypothesis (see Fig. 6)

Hypothesis 5. For any pair $\mathbf{V}_{l}, \mathbf{Q}_{l}^{(j)}$ there exists a vertex of $\Delta, \mathbf{V}_{l_{j}}$, such that $\mathbf{V}_{l}, \mathbf{Q}_{l}^{(j)}, \mathbf{V}_{l_{j}}$ are collinear.

If Hypothesis 5 holds, we can easily estimate

$$
\frac{1}{2}\left(\mathbf{Q}_{l}^{(j)}-\mathbf{V}_{l}\right) \nabla^{2} f\left(\mathbf{V}_{l}\right)\left(\mathbf{Q}_{l}^{(j)}-\mathbf{V}_{l}\right)^{T}
$$

by the second (univariate) divided difference of $f$ along $\mathbf{V}_{l} \mathbf{Q}_{l}^{(j)}$,

$$
\left\|\mathbf{V}_{l} \mathbf{Q}_{l}^{(j)}\right\|^{2}\left[\mathbf{V}_{l}, \mathbf{Q}_{l}^{(j)}, \mathbf{V}_{l_{j}}\right] f .
$$


This estimate is exact for any element of $\mathbb{P}_{2}$, and contains values of $f$ at $\mathbf{V}_{l}, \mathbf{Q}_{l}^{(j)}, \mathbf{V}_{l_{j}}$ only. Thus, from Theorem 2 we immediately have the following result

Theorem 6. If Hypothesis 5 holds, define

$$
\begin{gathered}
\mathcal{Q}_{2} f:=\sum_{l=1}^{N_{v}} \sum_{j=1}^{3} \mu_{l}^{(j)}(f) B_{l}^{(j)} \\
\mu_{l}^{(j)}(f):=f\left(\mathbf{Q}_{l}^{(j)}\right)-\left\|\mathbf{V}_{l} \mathbf{Q}_{l}^{(j)}\right\|^{2}\left[\mathbf{V}_{l}, \mathbf{Q}_{l}^{(j)}, \mathbf{V}_{l_{j}}\right] f .
\end{gathered}
$$

then

$$
\mathcal{Q}_{2} p=p, \forall p \in \mathbb{P}_{2} .
$$

Remark 7. It is immediate to verify that, when Hypothesis 5 holds, if we choose $\mathbf{W}_{l_{j}}:=\mathbf{V}_{l_{j}}, l=1, \ldots, N_{v}, j=1,2,3$ in (21) and $\zeta_{l}^{(j)}=\lambda_{l}^{(j)}$ in (21)-(22) then the q.i. defined by (9), (16), (21) and (22) coincides with (24).

Remark 8. The q.i. (24) reproduces quadratic polynomials and only uses linear combination of the values of the given function $f$ at the vertices of $\Delta$ or at points belonging to edges of the triangulation (or to their prolongation). However, it is reasonable to assume that the function $f$ can be evaluated at some (given) points belonging to the edges of $\Delta$, but it seems too restrictive to ask for values of $f$ at any point of the edges of $\Delta$. So, it can be useful to deal with quasi-interpolants where the evaluation points can be selected according to the available data. On this concern, if Hypothesis 5 holds, we can apply Theorem 4 with the choice

$$
\mathbf{W}_{l_{j}}:=\mathbf{V}_{l_{j}}, l=1, \ldots, N_{v}, j=1,2,3,
$$

and selecting $\zeta_{l}^{(j)}$ so that $\mathbf{Z}_{l}^{(j, 2)}$ in (21) does not necessarily coincides with $\mathbf{Q}_{l}^{(j)}$ but is a point where the value of $f$ is available.

We end this Section by noting that from the Hermite interpolant, (see (9), (11) and (8)), using the same approach as in Theorem 6, it is not difficult to construct a q.i. which reproduces $\mathbb{P}_{2}$ and only uses values of the given function $f$. It suffices to approximate $\nabla f\left(\mathbf{V}_{l}\right)$ by means of a linear combination of values of $f$ which provides an exact estimate for quadratic polynomials. As an example, if $\mathbf{S}_{l}^{(1)}, \mathbf{S}_{l}^{(2)}$ are two points not collinear with $\mathbf{V}_{l}$ we can estimate the directional derivative

$$
\nabla f\left(\mathbf{V}_{l}\right)\left(\mathbf{S}_{l}^{(k)}-\mathbf{V}_{l}\right)^{T}, k=1,2,
$$

by

$$
d_{l}^{(k)}:=\frac{f\left(\mathbf{D}_{l}^{(k)}\right)+\nu_{l, k}\left(\nu_{l, k}-2\right) f\left(\mathbf{V}_{l}\right)-\left(1-\nu_{l, k}\right)^{2} f\left(\mathbf{S}_{l}^{(k)}\right)}{\nu_{k, l}\left(1-\nu_{k, l}\right)}
$$


where

$$
\mathbf{D}_{l}^{(k)}:=\nu_{k, l} \mathbf{V}_{l}+\left(1-\nu_{k, l}\right) \mathbf{S}_{l}^{(k)}, \nu_{k, l} \neq 0,1
$$

From (25) we immediately obtain an estimate for the gradient of $f$ which can be substituted to $\nabla f\left(\mathbf{V}_{l}\right)$ in (11) providing the following new expression for the coefficients of the q.i. (9)

$$
\left(\begin{array}{c}
\mu_{l}^{(1)}(f) \\
\mu_{l}^{(2)}(f) \\
\mu_{l}^{(3)}(f)
\end{array}\right):=M_{l}^{-1}\left(\begin{array}{ccc}
1 & 0 & 0 \\
0 & S_{l, x}^{(1)}-V_{l, x} & S_{l, y}^{(1)}-V_{l, y} \\
0 & S_{l, x}^{(2)}-V_{l, x} & S_{l, y}^{(2)}-V_{l, y}
\end{array}\right)^{-1}\left(\begin{array}{c}
f\left(\mathbf{V}_{l}\right) \\
d_{l}^{(1)} \\
d_{l}^{(2)}
\end{array}\right)
$$

Since estimates (25) of the directional derivatives are exact for quadratic polynomials, the q.i. (9) with coefficients given by (26) reproduces $\mathbb{P}_{2}$. It is worthwhile to note that the coefficients of the obtained q.i. are not of the form (16) because each coefficient consists in a linear combination of five values of $f$.

In order to minimize the number of needed values of $f$, it is convenient to select the points $\mathbf{S}_{l}^{(k)}$ as vertices of $\Delta$.

On the other hand, since any element of $\mathcal{S}_{2}^{1}\left(\Delta_{P S}\right)$ is a quadratic polynomial in each subtriangle of the Powell-Sabin refinement of $\Delta$, if all the five points

$$
\mathbf{V}_{l}, \mathbf{S}_{l}^{(1)}, \mathbf{S}_{l}^{(2)}, \mathbf{D}_{l}^{(1)}, \mathbf{D}_{l}^{(2)},
$$

belong to the same subtriangle of the Powell-Sabin refinement of $\Delta$ we have:

$$
\left(\begin{array}{cc}
S_{l, x}^{(1)}-V_{l, x} & S_{l, y}^{(1)}-V_{l, y} \\
S_{l, x}^{(2)}-V_{l, x} & S_{l, y}^{(2)}-V_{l, y}
\end{array}\right)^{-1}\left(\begin{array}{c}
d_{l}^{(1)} \\
d_{l}^{(2)}
\end{array}\right)=\nabla^{T} f\left(\mathbf{V}_{l}\right), \forall f \in \mathcal{S}_{2}^{1}\left(\Delta_{P S}\right)
$$

Thus we can state the following:

Theorem 9. Let $\mathcal{Q}$ be any q.i. of the form (9) with $\mu_{l}^{(j)}$ defined according to (26). If all the five points $\mathbf{V}_{l}, \mathbf{S}_{l}^{(1)}, \mathbf{S}_{l}^{(2)}, \mathbf{D}_{l}^{(1)}, \mathbf{D}_{l}^{(2)}$ belong to the same subtriangle of the Powell-Sabin refinement of $\Delta$ then

$$
\mathcal{Q} s=s, \quad \forall s \in \mathcal{S}_{2}^{1}\left(\Delta_{P S}\right)
$$

\section{$\S 5$. Computed Examples}

In this Section we illustrate with two examples the performances of the quasi-interpolating schemes presented above.

Before presenting the mentioned examples we note that, in order to construct in practice the quasi-interpolants analyzed in Theorem 6 and in Remark 8, we have to produce a family of B-splines whose associated 

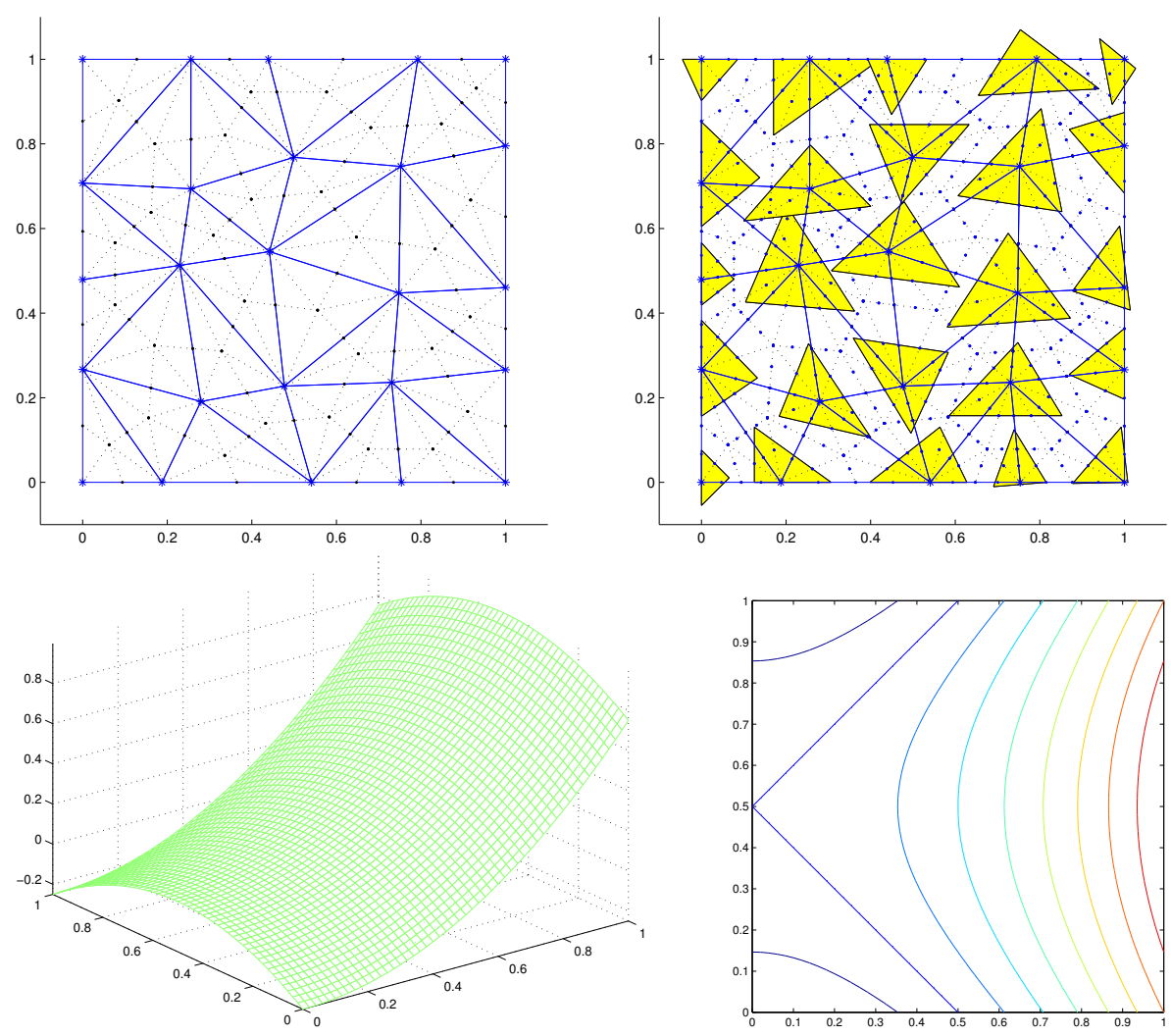

Fig. 7. Example 1. Top: triangulation and triangles determining the PowellSabin B-splines. Bottom: $\tilde{\mathcal{Q}}_{2} f$ and its level sets.

points $\mathbf{Q}_{l}^{(j)}$ verify Hypothesis 5. In order to obtain this, we construct for any vertex $\mathbf{V}_{l}$ of the triangulation $\Delta$ the three associate B-splines

$$
B_{l}^{(1)}, B_{l}^{(2)}, B_{l}^{(3)},
$$

by imposing that the vertices of the corresponding triangle, $\mathbf{Q}_{l}^{(1)}, \mathbf{Q}_{l}^{(2)}, \mathbf{Q}_{l}^{(3)}$, belong to some edges of $\Delta$ emanating from $\mathbf{V}_{l}$ (or to their prolongation). According to Section 2, to ensure positivity of functions (27) we have to impose that this triangle contains the Bézier points "surrounding" $\mathbf{V}_{l}$ (see Theorem 1 and Fig. 3). Moreover, in order to obtain a B-spline basis of $\mathcal{S}_{2}^{1}\left(\Delta_{P S}\right)$ with good performances from the computational point of view, we will determine the required triangle so that it has a small - possibly minimal - area, see Fig. 6.

Algorithm 10 summarizes the procedure we have used, for the interior vertices $\left\{\mathbf{V}_{l}, l=1, \ldots, N_{I}\right\}$, in order to obtain a "good" family of Bsplines so that Hypothesis 5 holds and positivity of the basis elements is ensured. Of course, the outlined procedure has to be suitably adapted for the boundary vertices. Moreover, we mention that, from the numerous numerical tests we performed, it turns out that other "initial" strategies 

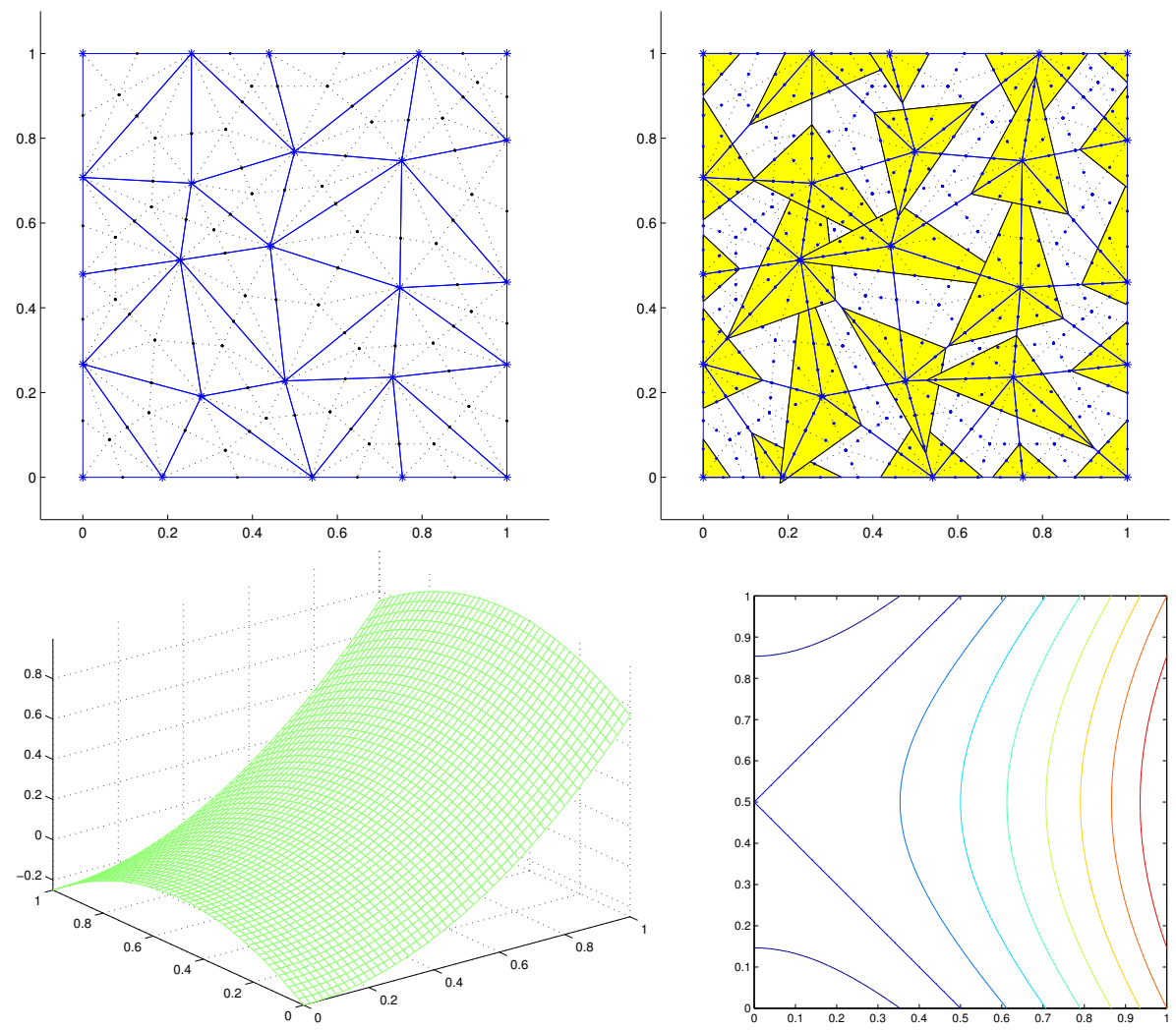

Fig. 8. Example 1. Top: triangulation and triangles determining the second set of Powell-Sabin B-splines. Bottom: $\mathcal{Q}_{2} f$ and its level sets.

can be sometimes efficiently used instead of that one described in step 2.1. For the sake of brevity we omit the related details.

\section{Algorithm 10.}

1. Let the triangulation $\Delta$ and the Powell-Sabin refinement $\Delta_{P S}$ be given;

2. for $l=1, \ldots, N_{I}$

2.1. compute the three $B$-splines $\hat{B}_{l}^{(j)}, j=1,2,3$ and the corresponding points $\hat{\mathbf{Q}}_{l}^{(j)}$ according to the minimal area procedure described in $[6]$;

2.2. for $j=1,2,3$ determine the projection $\tilde{\mathbf{Q}}_{l}^{(j)}$ of $\hat{\mathbf{Q}}_{l}^{(j)}$ onto the closest edge of $\Delta$ emanating from $\mathbf{V}_{l}$ (or onto its prolongation);

2.3. select a point $\mathbf{Q}_{l}^{(j)}$ over the line $\tilde{\mathbf{Q}}_{l}^{(j)} \mathbf{V}_{l}, j=1,2,3$, so that the triangle

$$
\mathbf{Q}_{l}^{(1)} \mathbf{Q}_{l}^{(1)} \mathbf{Q}_{l}^{(3)}
$$

has minimal area and contains all the Bézier points "surrounding" the vertex $\mathbf{V}_{l}$; 

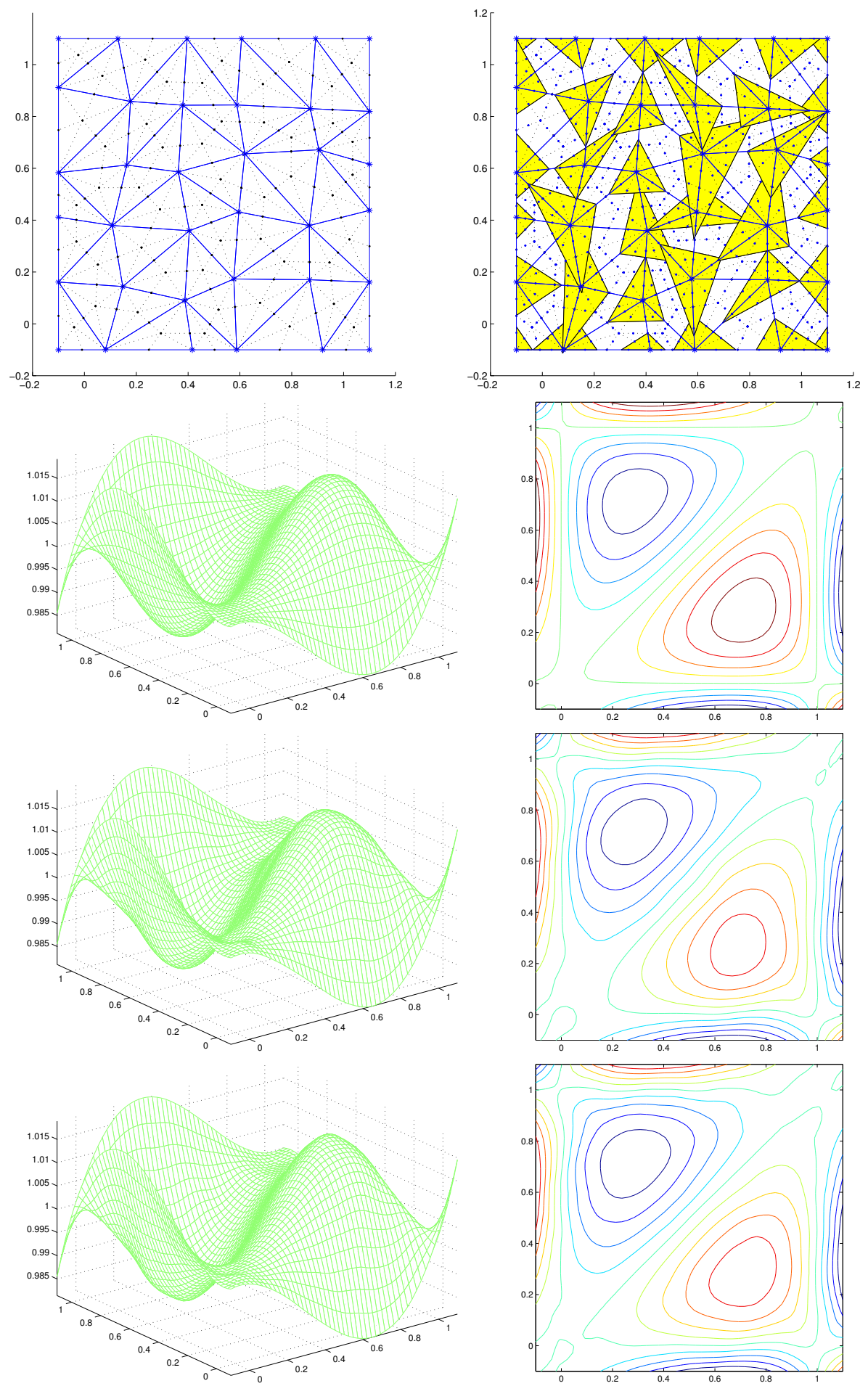

Fig. 9. Example 2. Top to Bottom: triangulation and triangles determining the set of Powell-Sabin B-splines; $f$ and its level sets; $\mathcal{Q}_{2} f$ and its level sets; the interpolant (9)-(11) and its level sets . 
2.4. compute the three $B$-splines $B_{l}^{(j)}, j=1,2,3$, corresponding to the the three points $\mathbf{Q}_{l}^{(j)}, j=1,2,3$.

In the first Example we have considered the quadratic polynomial

$$
f(x, y)=x^{2}-(y-0.5)^{2},
$$

in the unit square. The considered triangulation with its Powell-Sabin refinement is depicted in Fig. 7 top-left. Fig. 7 top-right shows the triangles determining a first set of Powell-Sabin B-splines computed according the minimal area procedure suggested in [6]. It can be seen as the vertices of the triangles do not lie in general on the edges of $\Delta$. Fig. 7 bottom shows the graph of $\tilde{\mathcal{Q}}_{2} f$ and its level sets. A perfect reproduction of $f$ is obtained.

Fig. 8 refers to the same function (28) over the same domain with the same triangulation and the same refinement $\Delta_{P S}$ but with a different set of B-splines. In such a case the B-splines are constructed in order to satisfy Hypothesis 5. The triangles determining the new set of Bsplines are depicted in Fig. 8 top-right: all the vertices of the triangles lie on the edges of $\Delta$ (or on their prolongations). Moreover, each triangle contains all the Bézier points "surrounding" the corresponding vertex so that positivity of the $B_{l}^{(j)}$ is ensured. Fig. 8 bottom shows the graph of $\mathcal{Q}_{2} f$ and its level sets. The quasi-interpolant does not require derivatives of $f$. Nevertheless, a perfect reproduction of $f$ is obtained.

In the second example we have considered the function

$$
f(x, y)=\exp (x(1-x)(1-y) y(x-y))
$$

Fig. 9 top shows the considered triangulation with the Powell-Sabin refinement and the triangles determining the used B-splines. All the vertices of the triangles lie on the edges of $\Delta$ (or on their prolongations). The second row of Fig. 9 shows the graph of $f$ and its level sets while in the third row we have the graph of $\mathcal{Q}_{2} f$ and its level sets. Finally, for comparison we depict in the last row of Fig. 9 the graph and the level sets of the the classical Powell-Sabin interpolant (see (9)-(11)). The two approximants are completely comparable and the contour line of $\mathcal{Q}_{2} f$ present a little bit smoother behaviour.

Acknowledgments. Work partially realized during the visiting of the first author to the Centre de Mathématiques, INSA de Rennes. The first author thanks INSA for the received support. 


\section{References}

1. de Boor, C., The quasi-interpolant as a tool in elementary polynomial spline theory, in Approximation theory I, H. Berens, E.W. Cheney, G.G. Lorentz, and L.L. Schumaker (eds.) Academic Press, New York, 1973 pp. 269-276.

2. Carnicer, J. M.; Dahmen, W., Convexity preserving interpolation and Powell-Sabin elements, Comput. Aided Geom. Design 9 (1992), 279289.

3. Chui, C. K.; Diamond, H.; Raphael, L., Shape-preserving quasiinterpolation and interpolation by box spline surfaces, J. Comput. Appl. Math. 25 (1989), 169-198.

4. Chen, G.; Chui, C. K.; Lai, M. J., Construction of real-time spline quasi-interpolation schemes. Approx. Theory Appl. 4 (1988), 61-75.

5. Dagnino, C.; Lamberti, P., Finite part integrals of local bivariate $C^{1}$ quasi-interpolating splines, Approx. Theory Appl. (N.S.) 16 (2000), $68-79$.

6. Dierckx, P., On calculating normalized Powell-Sabin B-splines, Comput. Aided Geom. Design 15 (1997), 61-78.

7. Laghchim-Lahlou, M.; Sablonnière, P., $C^{r}$-finite elements of PowellSabin type on the three direction mesh, Adv. Comput. Math. 6 (1996), 191-206.

8. Lai, M.-J.; Schumaker, L. L., Macro-elements and stable local bases for splines on Powell-Sabin triangulations, Math. Comp. 72 (2003), $335-354$.

9. Lamberti, P.; Manni, C., Tensioned Quasi-interpolation via Geometric Continuity, Adv. Comput. Math. 20 (2004) 105-127.

10. Lee, B.; Lyche, T.; Morken, K., Some Examples of Quasi-interpolants Constructed from Local Spline Projectors, in: Mathematical Methods for curves and Surfaces: Oslo 2000, T. Lyche and L.L. Schumaker (eds.) Vanderbilt University Press 2001, pp. 243-252.

11. Lorente-Pardo, J.; Sablonnière, P.; Serrano-Pérez, M. C., On the convexity of Powell-Sabin finite elements, in Advances in Computational Mathematics, Z. Chen, Y. Li;, C. A. Micchelli and Y. Xu (eds.), Lecture Notes in Pure and Appl. Math. 202, Dekker, New York, 19998, pp. 395-404.

12. Lyche, T.; Schumaker, L. L., Local spline approximation methods, J. Approximation Theory 15 (1975), 294-325.

13. Mulansky, B.; Schmidt, J. W., Powell-Sabin splines in range restricted interpolation of scattered data, Computing 53 (1994), 137-154. 
14. Powell, M. J. D.; Sabin, M. A., Piecewise quadratic approximations on triangles, ACM Trans. Math. Software 3 (1977), 316-325.

15. Sablonnière, P., Error bounds for Hermite interpolation by quadratic splines on an $\alpha$-triangulation, IMA J. Numer. Anal. 7 (1987), 495-508.

16. Sablonnière, P., Bernstein quasi-interpolants on [0,1], in Multivariate Approximation Theory, IV, C. K. Chui C.K., W. Schempp and K. Zeller, (eds.) ISNM 90, Birkhäuser-Verlag, Basel, 1989, pp. 287-294.

17. Sablonnière, P., Quasi-interpolants associated with $H$-splines on a three-direction mesh, in: Advanced Problems in Constructive Approximation, M. D. Buhmann and D.H. Mache (eds.), ISNM 142, BirkhäuserVerlag, Basel, 2002, pp. 187-201.

18. Schoenberg, I. J., Contribution to the problem of approximation of equidistant data by analytic functions. Part A. On the problem of smoothing or graduation. A first class of analytic approximation formulae, Quart. Appl. Math. 4 (1946), 45-99.

19. Willemans, K.; Dierckx, P., Surface fitting using convex Powell-Sabin splines, J. Comput. Appl. Math. 56 (1994), 263-282.

20. Windmolders, J., Powell-Sabin splines for Computer Aided Design, $\mathrm{PhD}$ Thesis University of Leuven (2003).

21. Windmolders, J.; Dierckx, P., Subdivision of uniform Powell-Sabin splines, Comput. Aided Geom. Design 16 (1999), 301-315.

Carla Manni

Dip. di Matematica - Università di Roma "Tor Vergata"

Via della Ricerca Scientifica, 00133 Roma, ITALY

manni@mat . uniroma2. it

Paul Sablonnière

INSA de Rennes, Centre de Mathématiques

20 avenue des Buttes de Coësmes

CS 14315, 35043-Rennes cdex , FRANCE

Paul.Sablonniere@insa-rennes.fr 